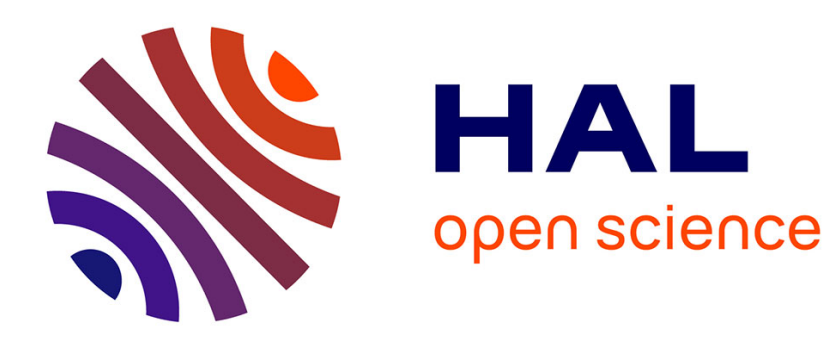

\title{
La régénération du référentiel d'une politique publique en faveur des sports de nature
}

Ludovic Falaix

\section{To cite this version:}

Ludovic Falaix. La régénération du référentiel d'une politique publique en faveur des sports de nature: L'étude de cas du département des Landes. Callède JP; Sabatier F; Bouneau C. Sport, Nature, et développement durable: une question de génération?, Maison des Sciences de l'Homme d'Aquitaine, pp.265-284, 2014, 978-2-85892-426-4. halshs-01015552

\section{HAL Id: halshs-01015552 \\ https://shs.hal.science/halshs-01015552}

Submitted on 4 Mar 2015

HAL is a multi-disciplinary open access archive for the deposit and dissemination of scientific research documents, whether they are published or not. The documents may come from teaching and research institutions in France or abroad, or from public or private research centers.
L'archive ouverte pluridisciplinaire HAL, est destinée au dépôt et à la diffusion de documents scientifiques de niveau recherche, publiés ou non, émanant des établissements d'enseignement et de recherche français ou étrangers, des laboratoires publics ou privés. 


\title{
La régénération du référentiel d'une politique publique en faveur des sports de nature? \\ L'étude de cas du département des Landes
}

Publié in Callède JP, Sabatier F, Bouneau C., 2014, Sport, Nature, et développement durable : une question de génération?, Pessac : Maison des Sciences de l'Homme d'Aquitaine, pp.265-284

\author{
Ludovic Falaix - ludovic.falaix@yahoo.fr \\ Maître de conférences \\ Université Blaise Pascal de Clermont-Ferrand \\ Laboratoire ACTé EA 4281
}

Les sports de nature, longtemps apanage de quelques élites sociales, pionnières de l'extrême, adeptes d'une «culture fun » (Lacroix, 1984¹), tendent à se démocratiser. Dans le département des Landes, les sports de nature connaissent même depuis plusieurs années un véritable engouement populaire. Le caractère essentiellement rural du département, la diversité du patrimoine naturel, la notoriété des vagues auprès des surfeurs, mais aussi la mobilisation du tissu associatif et du secteur marchand sont autant d'atouts qui participent à cette forme de démocratisation des sports de nature. Quelques chiffres illustrent cette dynamique ${ }^{2}$. En 2011, le département des Landes ne compte pas moins de 140 clubs sportifs proposant des activités physiques et sportives de nature et près de 10 000 licenciés évoluent au sein de ces structures ${ }^{3}$. Cette démocratisation qui dépasse le cadre landais soulève de nombreux enjeux sportifs, sociaux, économiques, environnementaux et spatiaux. Elle précipite l'émergence d'une politique publique élaborée par l'État au début des années 2000 dont le référentiel, c'est-à-dire «l'espace de sens qui [...] délimite des valeurs, des normes et des relations causales qui s'imposent comme cadre cognitif et normatif pour les acteurs »(Muller, 2005, 162), préconise d'agir en faveur «d'un développement maîtrisé » des sports de nature.

Cependant, l'État s'en remet aux départements auxquels il transfère l'exercice opérationnel d'une compétence sur ce segment des sports de nature ${ }^{4}$. Cet article envisage donc, à travers l'étude de cas du Conseil Général des Landes, la manière dont certaines collectivités locales s'approprient ce cadre cognitif et normatif qui consiste à garantir le développement maîtrisé des sports de nature.

\footnotetext{
${ }^{1}$ L'exigence intellectuelle induite par la publication scientifique invite à rappeler que les travaux de Gisèle Lacroix, construits autour du concept de "fun » pour caractériser l'esthétique et l'éthique sportive des populations glisse, sont antérieurs au succès éditorial sur la question d'Alain Loret.

${ }^{2}$ Il convient néanmoins d'appréhender ces chiffres avec précaution dans la mesure où un grand nombre de pratiquants ne sont pas affiliés auprès des instances fédérales. La lettre de l'économie du sport. « La pratique hors-club : danger ou opportunité ? », $\mathrm{n}^{\circ}$ 912, décembre 2008, 8 p.

3 Source : Comité Départemental Olympique et Sportif des Landes.

${ }^{4}$ Conformément à la loi no $84-610$ du 16 juillet 1984 modifiée le 6 juillet 2000 relative à l'organisation et à la promotion des activités physiques et sportives, les départements sont compétents pour intervenir sur le segment des sports de nature en favorisant leur développement maîtrisé.
} 
Le département des Landes, en invoquant le concept de développement maîtrisé des sports de nature, entend surtout mobiliser ces pratiques sportives comme de véritables vecteurs de développement touristique et territorial. Pour autant, ces orientations stratégiques qui traduisent une volonté d'émancipation des collectivités locales à l'idée de garantir le seul développement maîtrisé des sports de nature sont-elles des «opérations d'imposition de territorialité » (Violier, 1999 :17), l'expression d'une forme de régénération du référentiel de la politique publique conduite en ce domaine, et concourent-elles à l'émergence d'une identité territoriale (Michon, Terret, 2005) ? En examinant les jeux d'acteurs institutionnels et les processus décisionnels, cet article interroge donc la question d'un changement de référentiel de politique publique perceptible à travers les formes de mobilisation des sports de nature par les acteurs locaux.

En premier lieu, on reviendra sur la construction initiale par l'État du référentiel de politique publique et sur les mutations introduites par la Révision Générale des Politiques Publiques (RGPP) quant à la mise en œuvre opérationnelle du développement maîtrisé des sports de nature au sein des services déconcentrés. Fort de cet examen, on appréhendera alors le fait que l'appropriation d'une politique publique «sports de nature » par le Conseil Général des Landes répond davantage à une stratégie de marketing territorial qui prend corps à travers des outils de planification territoriale, la mise en scène "(géo-)graphique» (Debarbieux, 2003) des territoires et la promotion de manifestations sportives dessinant alors les contours d'un référentiel approprié par une génération d'acteurs locaux davantage enclins à dépasser l'injonction du développement maîtrisé des sports de nature au bénéfice du développement touristique et territorial.

\section{De l'émergence d'une politique publique en faveur des sports de nature au renouvellement des modalités d'engagement de l'État à l'heure de la révision générale des politiques publiques}

Les sports de nature ne «connaissent pas de définition arrêtée. Leur évolution permanente, l'apparition de nouvelles activités, comme la disparition d'autres, rendraient cette définition peu opérationnelle $e^{5} »$. Face au caractère aléatoire et évolutif des pratiques sportives de nature, le législateur, plutôt que de s'en remettre à une nomenclature des activités ou disciplines pratiquées, privilégie l'entrée par l'espace géographique et ses aménagements pour déterminer ce que recouvrent les sports de nature. Selon l'article L.311-1 du code du sport, "les sports de nature

\footnotetext{
${ }^{5}$ Ministère de la Jeunesse, des Sports et de la Vie Associative. Stat-Info, Bulletin de statistiques et d'études, les sports
} 
s'exercent dans des espaces ou sur des sites et itinéraires qui peuvent comprendre des voies, des terrains et des souterrains du domaine public ou privé des collectivités publiques ou appartenant à des propriétaires privés, ainsi que des cours d'eau domaniaux ou non domaniaux ». Cet article renforce donc l'acception générale qui s'appuie sur la notion "d'environnement spécifique », comme mentionné à l'article 43 de la loi sur le sport de 1984, pour procéder à une classification des activités physiques et sportives qui relèvent des sports de nature. D'autre part, le Ministère en charge des sports définit, dans l'instruction n04-131 JS du 12 août 2004, les sports de nature comme «les activités physiques et sportives dont la pratique s'exerce en milieu naturel, agricole et forestier - terrestre, aquatique ou aérien - aménagé ou non ». Aussi, face à la démocratisation des sports de nature et au regard des enjeux socio-territoriaux qu'ils recouvrent, l'État détermine les objectifs d'une politique publique.

\section{1 "Le développement maîtrisé des sports de nature" ou le référentiel d'une politique publique d'État en faveur des sports de nature}

Au début des années deux mille, les sports de nature acquièrent une certaine notoriété avec l'explosion du nombre de pratiquants rendue possible par la "technologisation » des activités. Le rapport établi en 2002 par le Conseil National des Activités Physiques et Sportive (CNAPS), présidé par Edwige Avice, ancien ministre, précise que «les sports de nature, véritables phénomènes des sociétés d'aujourd'hui, représentent une composante essentielle des politiques publiques d'aménagement et de développement des territoires ${ }^{6} »$. Plus encore, "vecteurs de développement touristique et de valorisation du milieu naturel, les sports de nature représentent en outre un enjeu local important en raison des retombées économiques et de la dimension éducative qu'ils sont susceptibles d'encourager et de générer ${ }^{7}$ ». Par conséquent, les pouvoirs publics décident de la mise en œuvre d'une réelle politique publique. En effet, à la demande du chef de l'État, JeanFrançois Lamour, alors Ministre de la Jeunesse, des Sports et de la Vie Associative, lance les États Généraux du Sport. Réunissant l'ensemble des acteurs du sport français, ces états généraux, lancés le 16 septembre 2002, permettent de définir de grandes orientations stratégiques quant à la promotion et la valorisation du sport en France. Parmi les objectifs déclinés à l'issue d'une large concertation, " permettre une meilleure appréhension de la dimension sports de nature » constitue une priorité ${ }^{8}$. En effet, les orientations retenues consistent à mieux encadrer l'offre et la demande

\footnotetext{
de nature en France en 2006, n08-01, avril 2008, p 1.

${ }^{6}$ CNAPS. Rapport au ministre des sports portant sur le bilan et les perspectives de développement des sports de nature, «Les sports de nature pour un développement durable », 2003, $122 \mathrm{p}$.

${ }^{7}$ Ministère de la Jeunesse, des Sports et de la Vie Associative. Stat-Info, Bulletin de statistiques et d'études, Les sports de nature en France en 2006, n08-01, avril 2008, p 1.

${ }^{8}$ Rapport publié conjointement par le Ministère de la Jeunesse, des Sports et de la Vie Associative et par le Comité
} 
des sports de nature. Elles doivent permettre «d'identifier et accompagner les problématiques liées à l'évolution des sports de nature afin de permettre de définir des cadres cohérents de pratiques; de valoriser un développement durable au sein des territoires par la mise en place de concertations départementales regroupant tous les acteurs concernés par les sports de nature; et de renforcer la concertation entre le mouvement sportif, les collectivités territoriales, les pratiquants et les pouvoirs publics ». Ces orientations sont par ailleurs établies à l'aune des grands principes avancés dans le Schéma des Services Collectifs du Sports (SSCS) dont la mise en œuvre opérationnelle devient effective avec la promulgation du décret $n^{\circ} 2002-560$ du 18 avril 2002. Fort de cette reconnaissance parlementaire et afin de renforcer son expertise, l'État se dote en 2003 d'un Pôle Ressource National des Sports de Nature (PRNSN), implanté au Centre Régional d'Education Populaire et de Sport (CREPS) de Vallon Pont d'Arc, haut lieu patrimonial naturel. Ce pôle a vocation à jouer le rôle de « tête de réseau » assurant ainsi un lien fonctionnel et opérationnel entre les services déconcentrés de la jeunesse, des sports et de la vie associative, les fédérations sportives, les associations et les collectivités territoriales. En 2007, l'État investit 533000 euros dévolus au fonctionnement du PRNSN et huit Équivalent Temps Plein (ETP) se consacrent à ces missions ${ }^{9}$.

Par ailleurs, une instruction, datée du 12 août 2004, $n^{\circ} 04-131$, ayant pour objet "l'intervention des services déconcentrés de la jeunesse et des sports dans le domaine des sports de nature » réaffirme l'engagement de l'État en matière « de promotion du développement maîtrisé des sports de nature ». L'instruction décline ce que recouvre la notion de développement maîtrisé et précise que l'État doit «favoriser l'accès des individus aux pratiques sportives de nature en sécurité et en améliorant leur compétence aux plans technique et environnemental et en s'appuyant sur des modes de pratiques diversifiés: associative, du secteur éducatif, du secteur marchand et contribuer à l'amélioration de la qualité des activités physiques et sportives qui s'exercent en milieu naturel. Cet objectif nécessite le développement de l'emploi qualifié, rémunéré et déclaré dans ce domaine sur la base d'une meilleure adéquation des qualifications actuelles aux métiers futurs, prenant encore mieux en compte l'évolution de la demande des pratique et l'émergence de nouvelles situations professionnelles ». Dans ce contexte, la mise en œuvre par l'État d'une politique publique sectorielle articulée autour de la question des sports de nature répond à double préoccupation et traduit la place des sports de nature dans la construction d'un rapport au monde (Muller, 2005). La première revêt une dimension sociale. Elle consiste à satisfaire à une demande citoyenne de pratique sportive s'exerçant en milieu naturel. L'enjeu est de renforcer la sécurisation des pratiques

National Olympique Français. «La mise en œuvre des mesures pour le développement du sports à l'issue des états généraux du sports », 2003, 40 p. 
sportives de nature en garantissant un encadrement professionnalisé. Les pratiques sportives de nature sont également présentées comme des leviers de socialisation, d'apprentissage des règles de vie collective, d'éducation citoyenne et à l'environnement. La seconde préoccupation est davantage en lien avec la notion de développement territorial durable. En effet, au regard de l'essor spectaculaire des sports de nature, des axes de développement territorial se déclinent tant sur le plan sportif que touristique, qu'environnemental, qu'économique ou social. L'ambition est alors de contenir l'essor spectaculaire et parfois anarchique des sports de nature en garantissant un encadrement des pratiquants et l'intégrité des qualités environnementales des espaces de pratiques. En revanche, l'État, auquel incombe, au delà de ses prérogatives régaliennes, des fonctions d'incitation, d'impulsion ${ }^{10}$ est désormais attentif à "faire-faire ». Car même si l'action de l'État dispose d'une présomption de légitimité, la délégation de compétence et de mise en œuvre d'une politique publique en faveur du développement maîtrisé des sports de nature est d'autant plus exacerbée avec la rationalisation des effectifs et des budgets induite par la révision générale des politiques publiques.

\section{2 «Faire-faire»: néo-paradigme de l'action publique d'État au sein des services déconcentrés ?}

La R.G.P.P. bouleverse la mise en œuvre des politiques publiques par les services déconcentrés dans le champ de la promotion et de la valorisation des sports de nature. La nouvelle organisation des directions interministérielles ${ }^{11}$ de la cohésion sociale doit "permettre de développer des modes d'intervention nouveaux, mieux adaptés à la diversité des situations à traiter, s'appuyant sur une expertise renforcée $e^{12} 》$. L'ingénierie sociale ${ }^{13}$ devient même le ressort paradigmatique des politiques publiques dont «l'élaboration consiste d'abord à construire une représentation, une image de la réalité sur laquelle on veut intervenir. C'est en référence à cette image cognitive que les acteurs organisent leur perception du problème, confrontent leurs solutions et définissent leurs propositions d'action : on appellera cette vision du monde le référentiel d'une politique » (Muller [1990], 2003 : 65). L'enjeu relève d'une acuité nouvelle à l'heure où le concept

\footnotetext{
${ }^{9}$ PRNSN. Rapport d'activité, année 2007, 6 p.

${ }^{10}$ Jacques Donzelot parle d'un "État animateur » évoquant, par là même, les difficultés des services déconcentrés à mettre en œuvre de manière pragmatique les politiques publiques que les administrations centrales impulsent (Donzelot, 1994).

${ }^{11}$ Pour obtenir la liste exhaustive des missions : consulter le décret $n^{\circ} 2009-1540$ du 10 décembre 2009 relatif à l'organisation et aux missions des directions régionales de la jeunesse, des sports et de la cohésion sociale, ainsi que le décret n²009-1484 du 3 décembre 2009 relatif aux directions départementales interministérielles.

${ }^{12}$ Circulaire du Premier Ministre du 19 septembre 2008 intitulée «Lancement de la phase de préfiguration des nouvelles Directions Régionales de la Jeunesse, des Sports et de la Cohésion Sociale (DRJSCS) ».

${ }^{13}$ La circulaire du Premier Ministre du 19 septembre 2008 relative à la création des DRJSCS emploie le terme d'ingénierie sociale pour désigner le champ des missions de ces nouvelles directions régionales.
} 
d'ingénierie sociale est mobilisé par les intellectuels chargés d'impulser la RGPP (Falaix, 2010). Les agents sont invités à s'en emparer pour garantir l'efficience des politiques publiques. En effet, la synthèse d'un groupe de travail sur l'ingénierie sociale décline les enjeux liés à l'application de ce concept. Celui-ci est ainsi «fortement reliée à l'émergence de la notion de gouvernance, définie comme un nouveau mode d'intervention de l'État, qui fait appel à la coopération des acteurs, à leur interaction pour la co-construction d'actions publiques. Autrement dit, l'État ne doit plus faire mais faire-faire ». Néanmoins, à l'heure où le gouvernement demande à ses agents de "faire-faire ", quel est le positionnement de ceux en charge du développement maîtrisé des sports de nature au sein des services déconcentrés?

Compte tenu des injonctions gouvernementales, "les référents sports de nature » des services déconcentrés ne peuvent plus faire l'économie d'un travail partenarial avec les collectivités territoriales d'autant plus que celles-ci disposent de compétences légales en matière de développement des sports de nature. En effet, la loi n84-610 du juillet 1984, dite « loi Avice », du nom du ministre de l'époque, modifiée le 6 juillet 2000 relative à l'organisation et à la promotion des activités physiques et sportives, confie la responsabilité aux départements de mettre en place une commission et un plan départemental des espaces, sites et itinéraires relatifs aux sports de nature. La loi précise que « le département favorise le développement maîtrisé des sports de nature. A cette fin, il élabore un plan départemental des espaces, sites et itinéraires relatif aux sports de nature... Il est institué une commission départementale des espaces, sites et itinéraires relatifs aux sports de nature, placée auprès du président du conseil général. Cette commission propose le plan départemental des espaces, sites et itinéraires relatifs aux sports de nature et concourt à son élaboration; propose les conventions relatives au plan ; est consultée sur toute modification du plan ainsi que sur tout projet d'aménagement ou mesure de protection des espaces naturels susceptibles d'avoir une incidence sur l'exercice des sports de nature dans les espaces, sites et itinéraires inscrits à ce plan. La composition et les modalités de fonctionnement de la commission sont fixées par délibération de l'assemblée départementale ". Se dessinent là les contours d'une injonction paradoxale à laquelle se confrontent les cadres de la fonction publique d'État exerçant au cœur des services déconcentrés. En effet, d'une part, l'État souhaite impulser une politique nationale de développement maîtrisé des sports de nature mais d'autre part, l'exercice opérationnel de cette compétence incombe exclusivement aux conseils généraux, seuls aptes à entériner la mise en œuvre du PDESI et à embrasser cette volonté étatique d'agir en faveur du développement maîtrisé. Plusieurs entretiens réalisés auprès du correspondant départemental des sports de nature à la Direction Départementale de la Cohésion Sociale et de la Protection des Populations (DDCSPP) des 
Landes, permettent de corroborer cette analyse. Celui-ci reconnaît volontiers que «l'État ne peut être qu'un incitateur dans le cadre de la mise en ouvre du PDESI ${ }^{14}$ ». D'autre part, ce professeur de sport admet que «l'État ne dispose d'aucun levier financier pour impulser une quelconque politique en faveur du développement maîtrisé des sports de nature ». La lecture des objectifs fixés, en 2011, dans le cadre des Budgets Opérationnels de Programmes (BOP) et des indicateurs de performance dans les champs des sports est sans appel : ceux consacrés au développement maîtrisé des sports de nature sont portion congrue ${ }^{15}$. En effet, dans le Programme 219 - Sports de 2011, jamais le développement maîtrisé des sports de nature n'est mentionné parmi les objectifs. Ainsi, l’État se recentre sur ses prérogatives régaliennes et s'éloigne quelque peu de son dessein de promouvoir, en qualité d'acteur et en y consacrant une part importante de ses crédits, une politique publique en faveur du développement maîtrisé des sports de nature. Certains évoquent alors l'idée que l'État se retire des territoires et gouverne désormais à distance. Ils précisent que les réformes administratives transforment le mode de conception et de mise en œuvre des politiques de l'État «en donnant au parlement et aux administrations centrales un rôle prépondérant dans la définition des programmes de l'État, avec pour effet corollaire la restriction des capacités d'adaptation des objectifs et des mesures dont disposaient jusque-là les services déconcentrés» (Epstein, 2005: 108). Par conséquent, si l'État impulse et clarifie les grandes orientations stratégiques en matière de développement maîtrisé des sports de nature, force est de constater qu'il appartient avant tout aux départements de proposer une déclinaison opérationnelle d'une politique publique en faveur du développement maîtrisé des sports de nature.

\section{La promotion des « sports de nature » au Conseil Général des Landes : vers la régénération du référentiel de politique publique}

En 2005, l'assemblée délibérante du Conseil Général des Landes statue en faveur de la création d'une Commission Départementale des Espaces, Sites et Itinéraires (CDESI) « destinée à recenser les sites afin de développer la pratique des sports de pleine nature ${ }^{16} »$. La CDESI se réunit pour la première fois le 21 juin $2006^{17}$ à l'hôtel du département. Elle confie, par une procédure

\footnotetext{
${ }^{14}$ Entretiens réalisés en mars/avril 2010 à la DDCSPP des Landes.

${ }^{15}$ Ministère du Budget. Rapport sur l'évolution de l'économie nationale et sur les orientations des finances publiques tome 2, juin 2011, p 85.

${ }^{16}$ Extrait de la délibération $n^{\circ} \mathrm{H} 6$ du 31 janvier 2005. Objet : les sports, $10 \mathrm{p}$.

${ }^{17}$ A l'heure où le Conseil Général des Landes institue sa propre CDESI, un bilan de l'état d'avancement des travaux sur les sports de nature dans les autres départements fait apparaître que 54 ont une CDESI initiée, 14 ont une CDESI instituée, 39 ont réalisé un état des lieux quant aux pratiques sportives de pleine nature, 3 disposent d'un PDESI opérationnel et 35 ne se sont pas encore emparés de la question. (Source : PRNSN).
} 
d'appel d'offres, à un prestataire la charge d'établir une étude-diagnostic sur les sports de nature dans les Landes afin d'affiner les résultats d'une enquête conduite en 2006 par le Comité Départemental du Tourisme (CDT) des Landes sur la question des activités sportives et de loisirs. Les objectifs de cette enquête visent à connaître en premier lieu le volume de fréquentation touristique de ces activités (nombre d'individus reçus, type de clientèle, dates d'ouvertures) et en second lieu le poids économique de ces activités. Les résultats de l'étude sont sans appel. Près de 1,3 millions de personnes sont reçues auprès de ces prestataires d'offre en termes d'activités sportives et de loisirs. Le chiffre d'affaire de ces structures représente plus de 15 millions d'euros. Plus de 1000 emplois concernent ce secteur d'activité au sein duquel le surf occupe une place privilégiée. En 2006, près de 50000 stagiaires sont accueillis au sein des 48 écoles de surf interrogées dans le cadre de l'enquête. Près de 3,5 millions d'euros sont générés par la seule activité surf. Par ailleurs, les résultats de l'enquête mettent en exergue la nécessité de renforcer la communication institutionnelle sur le patrimoine naturel landais comme support de pratique sportive de nature, d'améliorer les aménagements pour permettre la densification des pratiques sportives et récréatives, d'élargir les temps d'interventions de ces prestataires au delà de la période estivale $^{18}$ et d'accompagner le développement de certaine filière sportive, notamment la filière $\operatorname{surf}^{19}$. Fort des résultats de l'enquête quant au potentiel en terme de développement territorial que recouvrent les sports de nature, le Conseil Général des Landes, sur proposition de la CDESI, lance donc un appel d'offre sur la réalisation d'une étude prospective dont les résultats doivent permettre d'établir un diagnostic et des préconisations à l'endroit des sports de nature. Cette étude doit mettre en lumière les perspectives de développement par filière dans le respect des principes sous-jacents au concept de développement durable et de proposer des actions opérationnelles permettant de témoigner de l'engagement du département des landes pour les sports de nature. Par ailleurs, l'étude entend parfaire « une connaissance de l'offre et de la demande des clientèles et des pratiquants et par une analyse des pratiques existantes, de mesurer et d'analyser les retombées économiques directes et indirectes générées par les activités à travers les impacts engendrés par une activité, une filière ou un évènement sur le territoire ainsi que l'impact des sports de nature sur l'attractivité du territoire $^{20} »$. Par conséquent, le projet politique est sans ambiguïté. On est loin d'agir en faveur du seul développement maîtrisé des sports de nature. Il consiste à mobiliser les sports de nature comme

\footnotetext{
${ }^{18}$ Sud-Ouest du 16 décembre 2006. « Les activités sportives et de loisirs passées à la loupe ». A la lecture de l'article, on y apprend que "le régime saisonnier avec l'hypertrophie estivale a pour conséquence une répartition bancale du travail: 44\% des professionnels voudraient voir développer l'avant-saison ».

${ }^{19}$ Stéphanie Vandecrux, Comité Départemental des Landes. Résultats de l'enquête 2006, activités sportives et de loisirs, 2006, 8 p.

${ }^{20}$ Conseil Général des Landes. Cahier des Clauses Particulières - CCP. «Etude-diganostic Sports de Nature dans le département des Landes », 2006, 7 p.
} 
support d'une attractivité territoriale. Incontestablement les sports de nature sont présentés comme « un atout pour les territoires ${ }^{21}$ ». Ainsi, s'appuyant sur l'étude-diagnostic et à l'issue des travaux de concertation, les membres de la CDESI proposent à l'assemblée délibérante du Conseil Général des Landes l'adoption d'un Plan Départemental des Espaces, Sites et Itinéraires (PDESI), véritable outil de planification territoriale, dont les objectifs opérationnels témoignent de la manière dont cette génération d'acteurs locaux s'approprie la commande de l'État consistant à favoriser le développement maîtrisé des sports de nature. Une régénération du référentiel de politique publique en faveur des sports de nature s'opère.

\section{Les outils de planification territoriale axés sur la valorisation des sports de nature}

\subsection{Une inscription d'Espaces, Sites et Itinéraires structurants dont l'initiative revient aux acteurs locaux}

Outil de planification, le PDESI des Landes s'articule autour de plusieurs objectifs au sein desquels figure en premier lieu "le renforcement de l'offre en termes de pratique sportive ${ }^{22}$ ». Néanmoins, le PDESI doit également permettre "d'inscrire de nouveaux Espaces, Sites et Itinéraires afin de pérenniser les pratiques existantes, de développer de nouvelles pratiques en garantissant une gestion concertée des espaces ainsi qu'une valorisation environnementale et une accessibilité des lieux et des pratiques pour tous ». Ainsi, l'intérêt du PDESI est décliné en cinq axes. A travers ce plan, le Conseil Général des Landes entend «constituer pour le département un patrimoine et une vitrine de sites de pratiques et garantir à l'usager leur accessibilité, leur entretien, leur sécurité; valoriser et préserver son patrimoine naturel et de sensibiliser à l'environnement en y associant ses schémas départementaux des Espaces Naturels Sensibles et de Gestion et de Valorisation des cours d'eau; définir ses propres interventions, pour ce qui concerne l'acquisition, l'aménagement, l'entretien, la gestion et de façon plus générale la promotion des ESI landais; promouvoir et développer la pratique des sports de nature en favorisant tout autant l'accès aux différents espaces et sites existants et la conciliation des usages, afin de privilégier une attractivité équitable sur le territoire qui permettra de renforcer l'offre touristique landaise ». Pas une fois n'est décliné le développement maîtrisé des sports de nature au sein des cinq axes qui structurent le PDESI. Il est plutôt un outil au service du développement local et de l'attractivité

\footnotetext{
${ }^{21}$ «Sports de nature, un atout pour les territoires ». La gazette des communes, décembre 2008, n 46/1960, p 34-36.

${ }^{22}$ Plan Départemental des Espaces, Sites et Itinéraires. « Développement maîtrisé des sports de nature pour favoriser la pratique sportive des landais et contribuer à l'attractivité touristique du territoire tout en préservant et valorisant les qualités environnementales du département ». Conseil Général des Landes, avril 2011, 32 p.
} 
territoriale. Les Espaces, Sites et Itinéraires (ESI) à inscrire au PDESI relèvent d'une hiérarchisation en fonction du niveau de l'échelle territoriale de l'emprise foncière. L'ensemble des acteurs locaux issus de la sphère publique peuvent solliciter l'inscription d'un ESI au PDESI des Landes. Ainsi, le PDESI des Landes distingue :

«- ESI de niveau III: ESI d'intérêt départemental dans la mesure où les activités pratiquées sur ces lieux sont supports à une politique de promotion et de mise en marché des activités sportives et présentent des incidences sportives et touristiques fortes auprès d'un large panel d'usagers.

- ESI de niveau II: ESI d'intérêt départemental de rang inférieur puisque seul le volet sportif est prépondérant. ESI qui supportent des pratiques sportives relevant d'un environnement spécifique et qui jouit d'une grande notoriété auprès des publics initiés

- ESI de niveau I: ESI d'intérêt local où s'observe une pratique sportive affirmée et régulière mais dont l'attractivité ne dépasse par l'échelon local ${ }^{23} »$.

Seule l'inscription d'un ESI de niveau III fait l'objet d'un accompagnement financier du Conseil Général des Landes ${ }^{24}$ afin de garantir un entretien, une gestion, un aménagement et une promotion de ces lieux. Cependant, force est de constater que l'initiative des inscriptions des ESI appartient aux acteurs locaux qu'ils soient structure associative, collectivité territoriale ou établissement public. Par conséquent, la mise en œuvre du PDESI des Landes, qualifié de " sélectif», dépend étroitement du degré d'implication des acteurs locaux dans l'appropriation du projet politique. D'autre part, le PDESI est mobilisé comme un outil de planification territoriale qui privilégie les aménagements sur les seuls ESI de niveau III. Incombent, au demeurant, aux collectivités locales d'adapter leurs propres outils de planification territoriale (SCOT, PLU) afin de reconnaître localement la classification de ces espaces consacrés aux sports de nature.

\subsection{Le PDIPR et le renforcement de l'offre touristique locale}

La loi du 22 juillet 1983 relative à la répartition de compétences entre les communes, les départements, les régions et l'État confie aux départements la mise en œuvre d'un Plan

\footnotetext{
${ }^{23}$ Rapport du Président. «Budget primitif 2011. Examen public des dossiers les 14-15 avril 2011, Volume 2 ». Délibération H6 - Direction de l'Education, de la Jeunesse et des Sports, Sports de nature, adoption PDESI, p. 895-939.

${ }^{24}$ Le département des Landes mobilise la Taxe Départementale des Espaces Naturels Sensibles (TDENS). La TDENS est instituée depuis le $1^{\text {er }}$ avril 1991 (Délibération $n^{\circ} \mathrm{F} 4$ du 31 janvier 1991) et s'applique à hauteur d'un taux établi à 2\%. La loi du 23 février 2005 relative au développement des territoires ruraux précise que la TDENS peut être mobilisée " pour des opérations d'acquisition, d'aménagements et de gestion des espaces, sites et itinéraires figurant au PDESI relatif aux sports de nature, sous réserve que l'aménagement ou la gestion envisagés maintiennent ou
} 
Départemental des Itinéraires de Promenade et de Randonnée non motorisée (PDIPR). A cet égard, les services de la direction de l'environnement du Conseil Général des Landes, en lien étroit avec le Comité Départemental du Tourisme, engagent, dans le cadre du PDIPR, une politique de développement des itinéraires pour la randonnée et le cyclable. En effet, en 2011, la direction de l'environnement propose une aide à la création et la restauration d'itinéraires de promenades thématiques afin de diversifier l'offre existante en complétant les itinéraires inscrits dans le PDIPR qui représentent aujourd'hui près de $3000 \mathrm{~km}$ de circuits balisés à l'attention des randonneurs pédestres, équestres et cyclistes. Cette aide à la création de boucle de promenades a essentiellement pour but de développer une offre locale avec l'identification d'un départ des itinéraires situé au cœur des centre-bourgs et dont les temps de parcours ne doivent pas excéder deux heures de marche. D'autre part, l'aide est susceptible d'être octroyée pour les opérations visant à la création et la restauration d'itinéraires de promenade thématique ciblés pour un public familial. Ces parcours thématiques, support d'une découverte du territoire local, doivent être en lien avec le patrimoine naturel et/ou culturel et des espaces didactiques et pédagogiques doivent jalonner les parcours. Ils privilégient le cheminement sur des espaces fonciers publics ou, à défaut, empruntent des parcelles privées pour lesquelles une convention d'autorisation de passage doit être co-signée entre la commune ou l'ONF et le propriétaire. Ainsi, la création de ces nouveaux espaces de randonnées relèvent donc à la fois d'une volonté de promouvoir la pratique sportive de nature et d'une politique de valorisation touristique. Ces espaces, même s'ils peuvent être supports à la pratique sportive, sont donc davantage des outils de découverte patrimoniale exercée en dilettante dans un cadre familial. Ainsi, de nouveaux espaces de randonnées pédestres, équestres et cyclables sont appelés à voir le jour et viendront renforcer l'offre sportive et touristique existante. A cet égard, l'État, à travers le Centre National pour le Développement du Sport (CNDS), conscient des enjeux sportifs et touristiques que revêtent les sentiers de randonnée dans les Landes a octroyé en 2009 au département une enveloppe de 325000 euros afin de procéder à la remise en état des itinéraires endommagés à l'issue du passage de la tempête Klaus ${ }^{25}$.

\subsection{Le Schéma départemental du tourisme et du thermalisme et la promotion de la filière surf}

Henri Emmanuelli, Président du Conseil Général des Landes, souligne que le «tourisme landais trouvera un nouveau souffle [s'il concourt à appréhender] le foncier comme une chance ; pour son attrait, bien sur, mais aussi pour la capacité qu'il procure d'accueillir de nouveaux

\footnotetext{
améliorent la qualité des sites, des paysages et des milieux naturels 》.

${ }^{25}$ Décision CNDS-DSE-DS, n²06 (SES n ${ }^{\circ} 6958$ ) du 28 juillet 2009. 3 p.
} 
projets et de mobiliser des partenaires exogènes ${ }^{26} »$. Ainsi, parmi les conditions qui autoriseraient l'émergence de ce nouveau souffle, la valorisation des grands espaces de nature constitue un moyen d'y parvenir. Trois axes déterminent les orientations stratégiques et se déclinent en autant «d'objectifs de taille XL pour devenir une vraie destination touristique ». Si l'axe un consiste à enclencher un nouveau cycle d'investissements à travers notamment la réalisation d'aménagements d'une station de nouvelle génération à Port d'Albret, la création d'un complexe résidentiel et touristique sportif à dominante golfique d'envergure internationale fonctionnant à l'année, c'est davantage l'axe deux du schéma qui retient l'attention. En effet, ce deuxième axe, intitulé «valoriser la pluralité des filières", propose de renforcer l'offre de tourisme de nature en valorisant le potentiel du tourisme vert et en renforçant la filière surf entendue comme un vecteur de "positionnement concurrentiel différenciateur ». L'affiche promotionnelle sur laquelle figure un surfeur sur une plage landaise, située dans le hall d'accueil de l'hôtel du département, témoigne de la mobilisation du surf pour affirmer la grandeur et le « caractère nature » du département et jouer sur le processus de différenciation territoriale. Elle témoigne de la mise en scène " (géo-)graphique de projet et de prospective de territoire " (Debarbieux, 2003). Quant au troisième et dernier axe, il consiste à " s'adapter à l'e-tourisme et fédérer les acteurs». A ce sujet, la constitution de clubs de promotion participe de la déclinaison opérationnelle du troisième axe. En effet, "les clubs sont constitués par filière entre offices de tourisme, prestataires d'activités et hébergeurs. Ils sont animés par le CDT et élaborent en pleine concertation les plans d'action, de promotion et de communication annuels. Ils sont financés à part égale par le CDT et les partenaires ». En 2011, le «club surf» voit le jour et la valorisation de la pratique surf sous-tend donc un objectif qui dépasse le champ sportif. Philippe Jacquemain, Président de la Chambre de Commerce et d'Industrie des Landes, qualifie les enjeux liés à l'accompagnement du surf et des filières économiques qu'il entraîne dans son sillage: "Les activités liées aux sports de glisse ont généré la création d'une véritable filière économique qui s'est développée sur le territoire aquitain représentant un milliard et demi d'euros de chiffre d'affaires. Cette économie constitue un atout indéniable pour l'Aquitaine qui bénéficie des retombées des principaux spots et pôles économiques pour accentuer leur notoriété internationale. L'organisation de compétitions mondiales de surf, qui se déroulent chaque année sur notre côte, engendre des retombées économiques indirectes sur l'offre touristique, et constitue un formidable vecteur d'image véhiculant modernité, sport et nature ${ }^{27}$ ». La mise en œuvre du schéma départemental du tourisme et du thermalisme s'inscrit également en résonance avec les procédures de labellisation encouragées par la direction du tourisme du Conseil Général

\footnotetext{
${ }^{26}$ Conseil Général des Landes. «Schéma départemental du tourisme et du thermalisme. Vers un nouveau souffle », édito, juin 2010, p 2.
} 
des Landes qui accompagne financièrement les porteurs de projets. Ainsi, la Fédération Française de Surf, «dont la présence dans les Landes représente un atout en termes d'images et de dynamisme pour le territoire ${ }^{28} »$, est accréditée du label Qualité Tourisme. Initié lors du comité interministériel sur le tourisme du 9 septembre 2003, le label Qualité Tourisme entend développer les emplois et créer de la richesse. Ainsi, au-delà du seul développement maîtrisé des sports de nature, la promotion du surf comme pratique sportive soulève des enjeux d'ordre économique et permet d'affirmer une identité territoriale locale.

\section{Les sports de nature au service du marketing touristique et du renforcement du lien social}

\subsection{Le Raid XL : "Un raid nature et d'aventure pour tous "}

En 2010, le département des Landes lance la première édition d'un raid sportif et d'aventure dont les retombées doivent permettre de renforcer l'attractivité et de jouer sur le processus de différenciation territoriale. Les enjeux de cette épreuve sportive sont multiples. En effet, non seulement cette manifestation sportive doit encourager une découverte du patrimoine littoral landais mais elle doit aussi renforcer l'adhésion du mouvement sportif au projet politique visant à renforcer l'attractivité territoriale et touristique à travers la promotion des sports de nature. En avril 2010, la première édition du Raid XL voit le jour. Raid sportif organisé sur trois jours, cette manifestation sportive dédiée aux sports de nature est également un support pour la découverte des Landes. En effet, même si cette épreuve sportive poursuit l'objectif de promouvoir la pratique sportive de nature, le Raid XL a également vocation à promouvoir la «valorisation de l'offre touristique des Landes, à travers notamment la découverte de sites présentant un intérêt environnemental ${ }^{29}{ }^{2}$. Organisé en partenariat avec le mouvement sportif et les communes du littoral, le Conseil Général des Landes entend d'une part développer une synergie dans le domaine sportif et répondre aux besoins des nombreux pratiquants landais et d'autre part valoriser le potentiel de développement touristique sur le littoral. Par ailleurs, le Raid XL n'est pas exclusivement consacré au déroulement des épreuves sportives. En effet, de nombreuses initiations au surf sont proposées sous le parrainage d'Antoine Delpero, sacré champion du monde de longboard en 2009, censé véhiculer une image de réussite sportive. Enfin, cette manifestation doit également permettre une valorisation des acteurs locaux issus du monde des sports. En effet, à travers ce raid, le Conseil Général et ses partenaires du mouvement fédéral surf entendent « mettre en avant le travail effectué par les clubs landais » dans

\footnotetext{
${ }^{27}$ PNRSN. La lettre du réseau national des sports de nature, « L'œil de l'expert », $\mathrm{n}^{\circ}$ 68,juin 2011, p 1.

${ }^{28}$ Rapport du Président. «Budget primitif 2011, Examen public des dossiers les 14-15 avril 2011, Volume 2 ». Délibération H5 - Direction de l'Education, de la Jeunesse et des Sports - Favoriser la pratique des sports, 2011 , p. 891.
} 
la mesure où «ces derniers fonctionnent toute l'année et assument pleinement leur rôle éducatif en direction des plus jeunes de leur territoire : découverte et apprentissage du surf évidemment, mais également sensibilisation aux comportements éco-responsables dans le cadre des projets EcoSurf $f^{30}$ véhiculés par la Fédération Française de Surf et le Comité Départemental de Surf des Landes ${ }^{31}$ ". Le Raid XL est également mobiliser afin de renforcer le lien social. Cet objectif est assigné dans le PDESI des Landes dans la mesure où le plan départemental entend « renforcer la cohésion sociale [puisque] toute activité s'inscrivant dans un cadre de rencontre de relations humaines développe des facteurs de lien social [...] et favorise l'affirmation de la personnalité, l'épanouissement, la santé, le respect et la convivialité. En cela, les activités physiques de pleine nature sont fédératrices et génératrices de ce lien social ${ }^{32} »$. Sans doute est-ce la raison pour laquelle on peut également lire que « les amateurs de sports de glisse ou nautiques trouvent sur la côte, les lacs et les rivières, des vagues, du vent, mais aussi une offre citoyenne ${ }^{33} »$. Par conséquent, force est de constater que les sports de nature sont mobilisés par le Conseil Général des Landes à des fins de renforcement du lien social, du vivre ensemble, de l'acquisition d'une conscience citoyenne.

\subsection{Quand « Le printemps des Landes » fait la part belle aux sports de nature}

Ce raid s'inscrit également au cœur d'une manifestation d'envergure départementale, intitulée « $L e$ printemps des Landes », dont l'initiative revient au Comité Départemental du Tourisme des Landes. Le surf et les activités physiques de pleine nature y ont la part belle et sont identifiées comme une opportunité pour ceux qui s'y adonnent d'acquérir "l'étoffe d'un héros ${ }^{34}$ ". "Le printemps des Landes » permet la mise en réseau des animations proposée à l'échelle locale. L'ensemble des Offices de Tourisme participent à la manifestation et constitue les relais locaux pour le développement des projets. A cet égard, Hervé Bouyrie, Maire de Messanges et Conseiller Général, rappelle que «l'objectif principal est d'étendre la saisonnalité, centrée généralement sur les mois de juillet et aô̂t. Les Landes peuvent également être un département attractif en période printanière. Ces manifestations sont l'occasion de prouver que les touristes peuvent y venir en période "hors saison". Elles attirent des Landais, des personnes des départements voisins mais

\footnotetext{
${ }^{29}$ Conseil Général des Landes. Dossier de presse. « Sports de nature et surf à l'honneur dans les Landes », $2010, \mathrm{p} 6$.

${ }^{30}$ "L'environnement des surfeurs, l'océan, est un milieu très fragile. Surfeurs, vous avez tout intérêt à montrer l'exemple si vous espérez pouvoir rider vos jolies vagues dans quelques années! Vous apprendre à mieux connaître votre terrain de sport, pour mieux le protéger, telle est la mission d'EcoSurf ». Fédération Française de Surf [En ligne], http://www.surfingfrance.com/commissions/eco-surf, [Page consultée le 12 avril 2011].

${ }^{31}$ Conseil Général des Landes. Dossier de presse. « Sports de nature et surf à l'honneur dans les Landes », $2010, \mathrm{p} 8$.

${ }^{32}$ Conseil Général des Landes. PDESI, 2011, p 20.

${ }^{33}$ XLandes magazine, $\mathrm{n}^{\circ} 16$, avril-mai 2011. « Dossier Sports de Nature », p 15.

34 Comité Départemental du Tourisme des $\quad$ Landes http://www.tourismelandes.com/fr/1244/pages/d/actualites-developpement/les-landes-font-le-plein-de-promos-auprintemps/page/0, [Page consultée le 22 mars 2011].
} 
aussi en provenance de région plus lointaine, comme l'Ile-de-France ${ }^{35}$ ». En 2010, la thématique retenue s'articule autour des animations sur l'eau. A titre d'exemple, des événements de nature différente sont mis en place autour du surf, des lacs, de la plongée. Cette thématique, assène l'élu local, " nous permet de faire découvrir le patrimoine environnemental landais à travers ses filières d'excellence ». Manifestement, le surf compte donc parmi les filières d'excellence qu'il convient de valoriser. En 2011, l'opération est reconduite du 9 au 25 avril. Fondée sur la promotion du territoire à travers l'e-tourisme, cette manifestation recense l'intégralité des animations proposées à l'échelle locale. Ainsi, sur le site Internet du CDT des Landes, est évoqué le fait que "pour une escapade improvisée dans les Landes, les vacances de Pâques sont la période rêvée ». A en croire l'offre touristique, il y en aurait pour tous les goûts; que l'on soit en famille, en couple ou friand d'activités physiques et sportives. Plus encore, on nous invite à nous interroger en ces termes : «Pourquoi ne pas profiter de ces offres pour vous initier au surf? Un week-end pour commencer et vous reviendrez cet été, en véritable champion peaufiner votre technique!». En guise de conclusion, une certitude se fait jour: "C'est certain, le Printemps des Landes vous offre l'étoffe d'un héros... printanier. A la sortie de l'hiver vous rêviez de vous ressourcer, de bons plans sympas... le Printemps des Landes l'a imaginé pour vous $!^{36} "$. Par conséquent, cette stratégie renforce l'offre touristique et participe de l'animation des territoires hors des périodes estivales. L’idée se concrétise et «Le Printemps des Landes » a vocation à étendre la période touristique audelà de la saison estivale afin « de passer d'un tourisme de cueillette à un tourisme de conquête $e^{37}$ ».

\section{Conclusion}

Les sports de nature sont donc mobilisés dans les Landes comme une véritable ressource territoriale (Gumuchian, Pecqueur, 2007) et le dessein politique dépasse largement le cadre cognitif et normatif assigné par l'Etat qui entendait que les conseils généraux favorisent et garantissent exclusivement leur développement maîtrisé. Au regard des liens que tissent les sports de nature avec les thématiques propres au développement économique et touristique, avec les enjeux liés à l'aménagement du territoire ${ }^{38}$, à l'éducation, l'insertion et/ou l'inclusion sociale par le sport ${ }^{39}$, la

${ }^{35}$ Propos d'Hervé Bouyrie. Conseil Général des Landes [En ligne], http://www.cg40.fr/1-28045-Detail-d-uneactualite.php?id_actualite $=265$, [Page consultée le 22 mars 2011].

36 Comité Départemental du Tourisme des Landes [En ligne], http://www.tourismelandes.com/fr/1244/pages/d/actualites-developpement/les-landes-font-le-plein-de-promos-auprintemps/page/0, [Page consultée le 22 mars 2011].

${ }^{37}$ Propos d'Hervé Bouyrie, Conseiller Général, Président de la commission tourisme, in XLandes magazine, ${ }^{\circ} 16$, avrilmai 2011, p 18.

${ }^{38}$ CNOSF et AMNYOS. «Le sports, acteur incontournable de l'aménagement du territoire : enjeux, expérimentation, outils, perspectives », 2003, 89 p.

${ }^{39}$ L'objectif intitulé " mettre le potentiel du sport au service de l'inclusion sociale, de l'intégration et de l'égalité des chances » est assigné dans le livre blanc sur le sport présenté par la commission européenne. Voir Commission Européenne. «Livre blanc sur le sport », 2007, 21 p. 
mise en œuvre dans les Landes d'une politique publique volontariste en faveur des sports de nature traduit des intentions qui dépassent la volonté d'en assurer le seul développement maîtrisé au bénéfice d'une construction territoriale. A l'heure où l'inscription des territoires dans une économie et une géographie de l'organisation (Veltz, 1996) s’impose comme une absolue nécessité pour conserver une forme de compétitivité dans le cadre de la mondialisation, il appartient aux acteurs locaux d'agir en faveur d'une différenciation territoriale. Sans doute est-ce la raison pour laquelle «dans les Landes, les sports de pleine nature constituent à la fois un atout pour les habitants du département et le tourisme ${ }^{40} »$. Cette forme de régénération du référentiel traduit alors des intentions politiques et une évolution dans la manière dont les problématiques et/ou potentialités liées aux sports de nature sont appréhendées.

En mobilisant le concept de référentiel «forgé pour rendre compte des politiques publiques comme production intellectuelle d'images sur la société » (Faure, Pollet, Warin, 1995, p. 10), une analyse cognitive des politiques publique en faveur des sports de nature permet donc d'appréhender "la médiation », c'est-à-dire "l'ensemble de ce processus de construction d'un rapport au monde » (Muller, 1995). Les choix arrêtés par le Conseil Général des Landes témoignent alors d'un réel projet territorial qui « $n$ 'est pas que le résultat, [qui] est le fondement de l'action publique, une empreinte réelle des pratiques sociales dans l'espace » (Vlès, 2001, p. 19). On voit donc, à travers l'exemple des sports de nature dans les Landes, comment les collectivités territoriales s'affranchissent parfois des préconisations étatiques dans l'exercice de leurs compétences transférées et se réapproprient de façon dynamique le référentiel. Si l'État définit le référentiel des politiques publiques, celui-ci, recentré sur ces fonctions régaliennes, laisse alors aux générations d'acteurs locaux l'opportunité de s'approprier les dogmes et le rapport au monde que sous-tend le référentiel. Peut-être est-ce la raison pour laquelle, en matière de sports de nature, le législateur privilégie une entrée laissant le champ suffisamment vaste aux interprétations afin que les acteurs locaux puissent s'approprier la notion de développement maîtrisé. N'en va-t-il pas là de l'efficience de la décentralisation dont l'ambition est de réhabiliter le local comme échelle d'intervention privilégiée dans le processus de développement touristique et territorial?

\footnotetext{
${ }^{40}$ Conseil Général des Landes [En ligne], http://www.cg40.fr/1-28836-Les-sports-de-nature.php, [Page consultée le 8 mars 2011].
} 


\section{Bibliographie}

-DEBARBIEUX Bernard, 2003, « Neuf enjeux de l'iconographie de projet et de prospective de territoire ", in DEBARBIEUX Bernard et LARDON Sylvie (dir.), Les figures du projet territorial, La Tour d'Aigues, Éditions de l'Aube, 272 p.

-DONZELOT Jacques, 1994, L'État animateur. Essai sur la politique de la ville, Paris, Éditions Esprit, 239 p.

-EPSTEIN Renaud, 2005, « Gouverner à distance. Quant 1'État se retire des territoires », Esprit, $\mathrm{n}^{\circ} 319,13 \mathrm{p}$.

-FALAIX Ludovic, 2010, «L'ingénierie sociale et territoriale dans les directions interministérielles de la cohésion sociale : vers une co-construction des politiques publiques entre cadres d'État et habitants », Carnets de géographes, $\mathrm{n}^{\circ} 1,14 \mathrm{p}$.

-FAURE Alain, POLLET Gilles, WARIN Philippe (dir.), 1995, La construction du sens dans les politiques publiques : débats autour de la notion de référentiel, Paris, L’Harmattan, 192 p.

-GUMUCHIAN Hervé, PECQUEUR Bernard, 2007, La ressource territoriale, Paris, Anthropos, $254 \mathrm{p}$.

-LACROIX Gisèle, 1984, «Les activités de pleine nature et le thème de "la glisse". Enjeux institutionnels, économiques et culturels : l'exemple de la planche à voile », Thèse de $3^{\text {ième }}$ cycle en Sociologie Générale, Université de Paris VII, 374 p.

-MICHON Bernard, TERRET Thierry (dir.), 2005, Pratiques sportives et identités locales, Paris, L'Harmattan, $431 \mathrm{p}$.

-MULLER Pierre, [1990], 2003, Les politiques publiques, Paris, PUF, 127 p.

-MULLER Pierre, 2005, «Esquisse d'une théorie du changement dans l'action publique : structures, acteurs et cadres cognitifs », Revue française de sciences politiques, vol $55, \mathrm{n}^{\circ} 1, \mathrm{p} .155$ 187.

-VELTZ Pierre, 1996, Mondialisation, villes et territoires, Paris, PUF, 288 p.

-VIOLIER Philippe (dir.), 1999, L'espace local et les acteurs du tourisme, Rennes, PUR, 177 p.

-VLES Vincent, 2001, Service public touristique local et aménagement du territoire, Paris, L'Harmattan, 222 p.

\section{Pour citer cet article}

FALAIX L. (2014). "La régénération d'une politique publique en faveur des sports de nature. L'étude de cas du département des Landes" in CALLEDE JP. SABATIER F. BOUNEAU C. (dir.), Sport, nature et développement durable : une question de génération ?, Pessac: MSHA, pp. 265-284. 